\title{
Elastography in pancreatic solid tumours diagnoses
}

\author{
Przemysław Dyrla ${ }^{1}$, Jerzy Gil ${ }^{1}$, Michał Florek ${ }^{1}$, Marek Saracyn²$^{2}$ Bartłomiej Grala ${ }^{3}$, Emil Jędrzejewski ${ }^{4}$, \\ Stanisław Wojtuń ${ }^{1}$, Arkadiusz Lubas ${ }^{2}$ \\ ${ }^{1}$ Department of Gastroenterology, Military Institute of Medicine, Warsaw, Poland \\ ${ }^{2}$ Department of Internal Medicine, Nephrology and Dialysis, Military Institute of Medicine, Warsaw, Poland \\ ${ }^{3}$ Department of Pathology, Military Institute of Medicine, Warsaw, Poland \\ ${ }^{4}$ Department of General Surgery, Oncology, Metabolic and Thoracic Surgery, Military Institute of Medicine, Warsaw, Poland
}

Prz Gastroenterol 2015; 10 (1): 41-46

DOI: $10.5114 /$ pg.2015.48994

Key words: endosonography, elastography, elasticity, strain ratio.

Address for correspondence: Przemysław Dyrla MD, PhD, Department of Gastroenterology, Military Institute of Medicine, 128 Szaserów St, 04-141 Warsaw, Poland, phone: +48 2268180 61, e-mail: pdyrla@wim.mil.pl

\begin{abstract}
Introduction: Pancreatic solid tumour diagnoses remain a challenge for modern medicine. However, using endosonography together with elastography helps to examine the elasticity of tissues and therefore may allow definition of the nature of pancreatic tumours.

Aim: To evaluate the usefulness of elastography with the strain ratio method and quantitative evaluation of pancreatic solid tumours.

Material and methods: A total of 54 patients with pancreatic solid tumours were treated with ultrasound endosonography with fine-needle aspiration biopsy. The control group contained 26 patients with normal pancreas. Pancreatic solid tumours and normal pancreas were analysed with elastography and elasticity evaluation of the interest area (A), reference (B), and the strain ratio factor (B/A). Postoperative histopathological or cytological examinations were the final diagnoses. Both postoperative and cytological diagnoses were compared with average elasticity parameters (A) and strain ratio factors (B/A).

Results: Average elasticity parameters $(A)$ and the strain ratio factors $(B / A)$ were: $0.025 \%(0.01-0.05 \%)$ for malignant process, and (B/A) 33.93 (18.23-75.45); (A) - 0.26\% (0.14-0.35\%), and (B/A) 5.35 (3.47-7.8) for inflammatory process; (A) $0.54 \%$ (0.35-0.82\%), and (B/A) 1.79 (1.02-2.05) for normal pancreatic tissue.

Conclusions: Malignant tumours have higher tightness factor compared to inflammatory tumours and normal pancreatic tissue. Elasticity parameters reach the highest levels in normal pancreatic tissue, lower in inflammatory tumours, and the lowest in malignant tumours.
\end{abstract}

\section{Introduction}

Pancreatic tumours constitute a real diagnostic problem, especially in the case of solid ones. In either case, diagnostic efficiency depends on the localisation, size, and echogenicity of the tumour. Pancreatic solid tumours can be malignant and benign. Inflammatory tumours in chronic pancreatitis, schwannomas, fibromas, haemangiomas, lipomas, and adenomas are all benign tumours. On the other hand, adenocarcinomas, neuroendocrinal neoplasms, solid pseudopapillary neoplasms, stromal tumours, lymphomas, as well as metastatic tumours are malignant. Ninety-five percent of pancreatic neoplasms come from the exocrine part of the pancreas, while the rest derives from the endocrine part. The most frequent malignant tumour is adenocarcinoma neoplasm, which makes up about $90 \%$ of all pancreatic tumours [1, 2]. However, the detection of solid pancreatic tumours is connected with several diagnostic problems. Differentiation between malignant or benign tumours, as well as the possibility of implementing surgical treatment, are the most important aspects in diagnosis. Radiological diagnosis of solid pancreatic tumours is of great importance all the way from detection of the tumour, through stage assessment, and choosing the method of treatment. At the moment, there is no perfect method of evaluating the malignancy and operating ability of tumours. Solid pancreatic tumours are usually detected accidentally during ultrasound examination of the abdomen. During the diagnostic process we use computed tomography (CT), magnetic resonance (MR), and endosonography (EUS) [3]. Endosonography is the best method for the detection of pancreatic solid tumours, and together with 
elastography, allows us to define the elasticity of the tissue and the character of solid pancreatic tumours.

\section{Aim}

The purpose of this study is to evaluate the usefulness of elastography with strain ratio and quantitative evaluation of pancreatic solid tumours.

\section{Material and methods}

In this study we examined 54 patients with pancreatic solid tumours, and 26 patients with normal pancreas, which were examined with EUS because of submucosal tumours of the upper gastrointestinal tract (Table I). Each of these persons was examined with EUS and computed tomography. Pancreatic solid tumours and normal pancreas were analysed with elastography and elasticity evaluation of the area of interest $(A)$, the reference area $(B)$, and the strain ratio factor $(B / A)$. Postoperative histopathological or cytological examinations were the final diagnoses, which were later compared with average elasticity parameters $(A)$ and strain ratio factors (B/A). The study was conducted in accordance

Table I. Patients of study group and control group

\begin{tabular}{lcc} 
Group & Number & Age [years] \\
\hline $\begin{array}{l}\text { Study: } \\
\text { Female }\end{array}$ & 20 & $40-85(67.7)$ \\
\hline Male & 34 & $30-73(55.4)$ \\
\hline $\begin{array}{l}\text { Control: } \\
\text { Female }\end{array}$ & 11 & $22-64(43.9)$ \\
\hline Male & 15 & $25-57(44.6)$
\end{tabular}

Table II. Histopathological/cytological evaluation

\begin{tabular}{lccc} 
& $\begin{array}{c}\text { Evaluation } \\
\text { after surgery }\end{array}$ & $\begin{array}{c}\text { Evaluation } \\
\text { of the biopsy }\end{array}$ & Together \\
\hline Malignant & 11 & 23 & 34 \\
\hline Inflammation & 3 & 17 & 20 \\
\hline Together & 14 & 40 & 54
\end{tabular}

Table III. Evaluation of elasticity (A) and strain rate ratio $(B / A)$

\begin{tabular}{lcc} 
& A & B/A \\
\hline Pancreatic cancer & $\begin{array}{c}0.025 \% \\
(0.01-0.05 \%)\end{array}$ & $\begin{array}{c}33.93 \\
(18.23-75.45)\end{array}$ \\
\hline $\begin{array}{l}\text { Inflammatory process } \\
\text { of the pancreas }\end{array}$ & $0.26 \%$ & 5.35 \\
\hline Normal pancreas tissue & $(0.14-0.35 \%)$ & $(3.47-7.8)$ \\
\hline & $0.54 \%$ & 1.79 \\
& $(0.35-0.82 \%)$ & $(1.02-2.05)$
\end{tabular}

with the Declaration of Helsinki and was approved by the Institutional Review Board as well by the Local Ethics Committees when required. All the patients provided informed consent.

\section{Results}

Malignant tumours were diagnosed in 11 cases and inflammatory tumours were diagnosed in 3 cases in histopathological/cytological diagnosis after surgical treatment. Malignant tumours were diagnosed in 23 cases, and inflammatory tumours were diagnosed in 17 cases after fine-needle biopsy. In 34 cases of pancreatic solid tumours we diagnosed malignant tumours: adenocarcinoma after surgical treatment $(n=9)$, neuroendocrine tumour after surgical treatment $(n=2)$, neoplasm cells after biopsy ( $n=23)$, chronic pancreatitis after biopsy $(n=17)$, and inflammatory tumour after surgical treatment $(n=3)$ (Table II). The average parameters with reference to cytological and histopathological results were fixed: (A) - 0.025\% (0.01-0.05\%), and (B/A) 33.93 (18.23-75.45) for adenocarcinoma, neuroendocrine tumour, and neoplasm cells; (A) - 0.26\% (0.14-0.35\%), and (B/A) 5.35 (3.47-7.8) for inflammatory process; (A) 0.54\% (0.35-0.82\%), (B/A) 1.79 (1.02-2.05) for normal pancreas tissue (Table III).

\section{Discussion}

Elastography is one of the methods that enable us to show real-time elasticity of the tissue. The result is shown as a red-yellow-green-blue coloured picture. This method is based on different elasticity and hardness of normal and pathological tissue [4]. Deformation ability of the tissue is then evaluated depending on the difference in ultrasonic waves received. In case of soft, normal tissue, which is more elastic, the difference in ultrasonic waves received is higher than in pathological, hard tissue. The deformation can be expressed in a five-level scale [5]: level 1 - green - normal tissue; level 2 - green, yellow and red - fibred tissue; level 3 - green and blue with blue supremacy resembling honeycomb - tissue with mixed hardness, level 4 - centre green and blue around - vascularised neoplasm tumours; level 5 - intensive blue - malignant neoplasm.

Elastography is most commonly used to diagnose breast pathological lesions, thanks to easy access to this organ [6]. Endosonography with an elastography option is the result of the rapid progress of ultrasound and endoscopic techniques. Elastography is a real-time examination and special software is needed. The coloured view from the examination is put in atypical $\mathrm{B}$-mode picture. During the examination, the region of interest is marked. As a result, there are two pictures on the screen - a typical ultrasound view and a view 
with a coloured elastogram [7]. As we know, elastography is one of the best methods in detecting pancreatic solid tumours. Combined EUS-FNA (fine-needle aspiration biopsy) method is characterised by high sensitivity and specificity in diagnosing pathological pancreatic lesions [8-11]. In a multi-trial study with 121 patients Giovannini et al. used EUS with elastography for the evaluation of pancreatic tumours [5]. Adenocarcinoma tumours were diagnosed in 72 cases, neuroendocrine tumours were diagnosed in 16 cases, metastatic tumours from other organs were diagnosed in 3 cases, and chronic pancreatitis was diagnosed in 30 cases. During this study Giovannini et al. used a five-level real-time elastography scale. The specificity of this method was evaluated at $80.6 \%$ and the sensitivity was $92.3 \%$. In another study Saftoiu et al. examined 68 patients with EUS and elastography and evaluated its usefulness in diagnosing pancreatic tumours. The observation period was 6 months. In 32 cases they diagnosed neoplasm tumours, chronic pancreatitis in 11 cases, neuroendocrine tumours in 2 cases, and normal pancreas in 22 cases. Special software was used to objectivise the results. The software allowed the doctors to describe the histogram of colours and elasticity of pathological tissue.

The sensitivity of this study was about $91.4 \%$ and the specificity was $88.9 \%$ [12]. In Iglesias-Garcia et al. the sensitivity of elastography was compared to the reference examination. The sensitivity was $100 \%$ and the specificity was $85.5 \%$ [13]. Hirche et al. showed different findings due to difficulties in defining the dimensions of the lesion. In the case of lesions bigger than $3.5 \mathrm{~cm}$ there was no possibility to examine the whole pathological lesion and a sufficient part of the surrounding normal tissue as a benchmark. That is why the sensitivity of the Hirche study was about $41 \%$ and the specificity was 53\% [14]. Notwithstanding, differentiation between chronic pancreatitis and pancreatic neoplasm remains a real challenge. Janssen et al. claim that elastography is not a precise examination to differentiate chronic pancreatitis and pancreatic cancer [15]. According to them, a histopathology examination is of utmost importance, and elastography only helps to choose the spot to make FNA and EUS [16]. One of the most interesting studies about using elastography in the differential diagnosis of pancreatic solid tumours

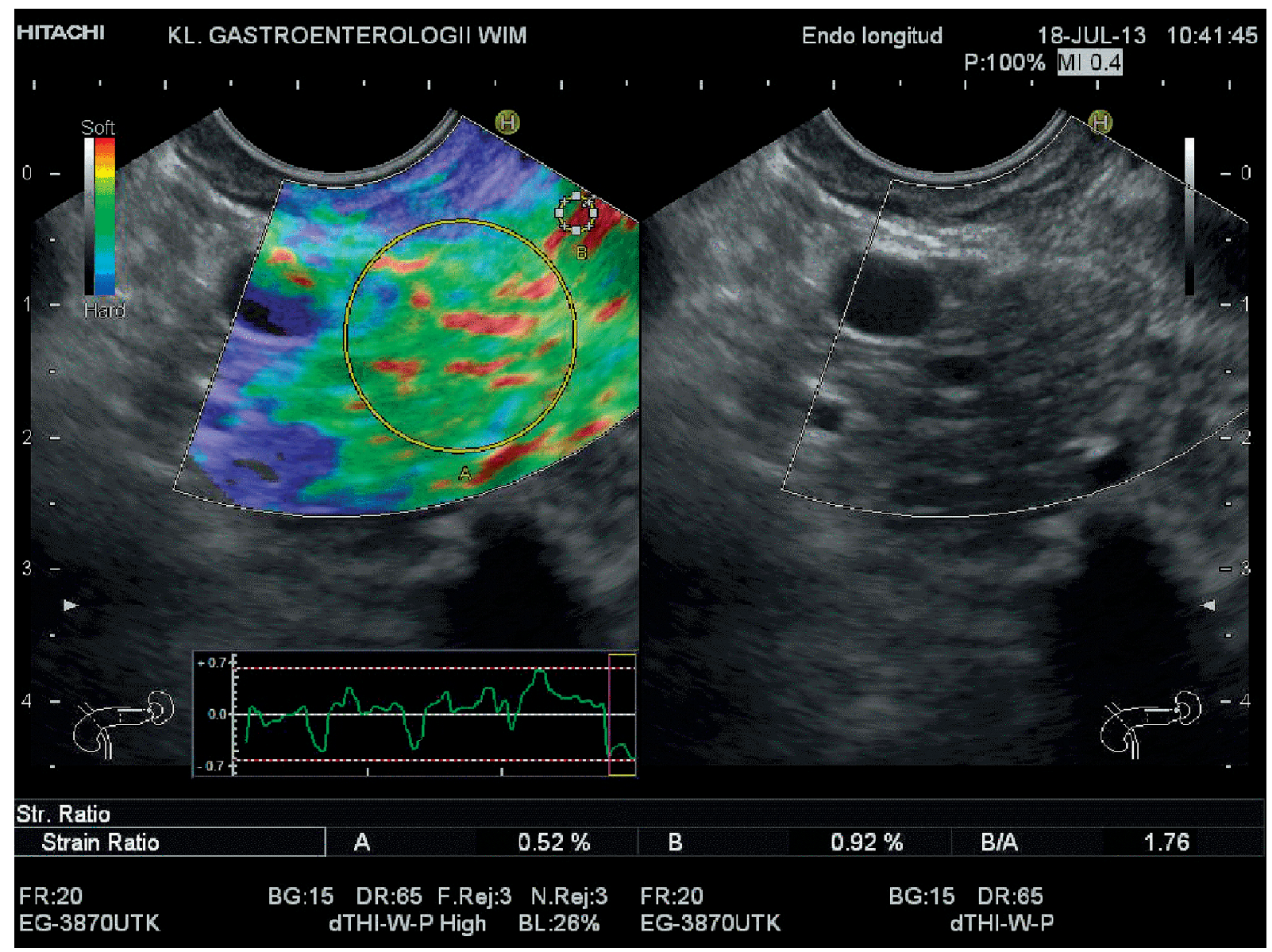

Figure 1. Normal pancreas tissue in elastography 


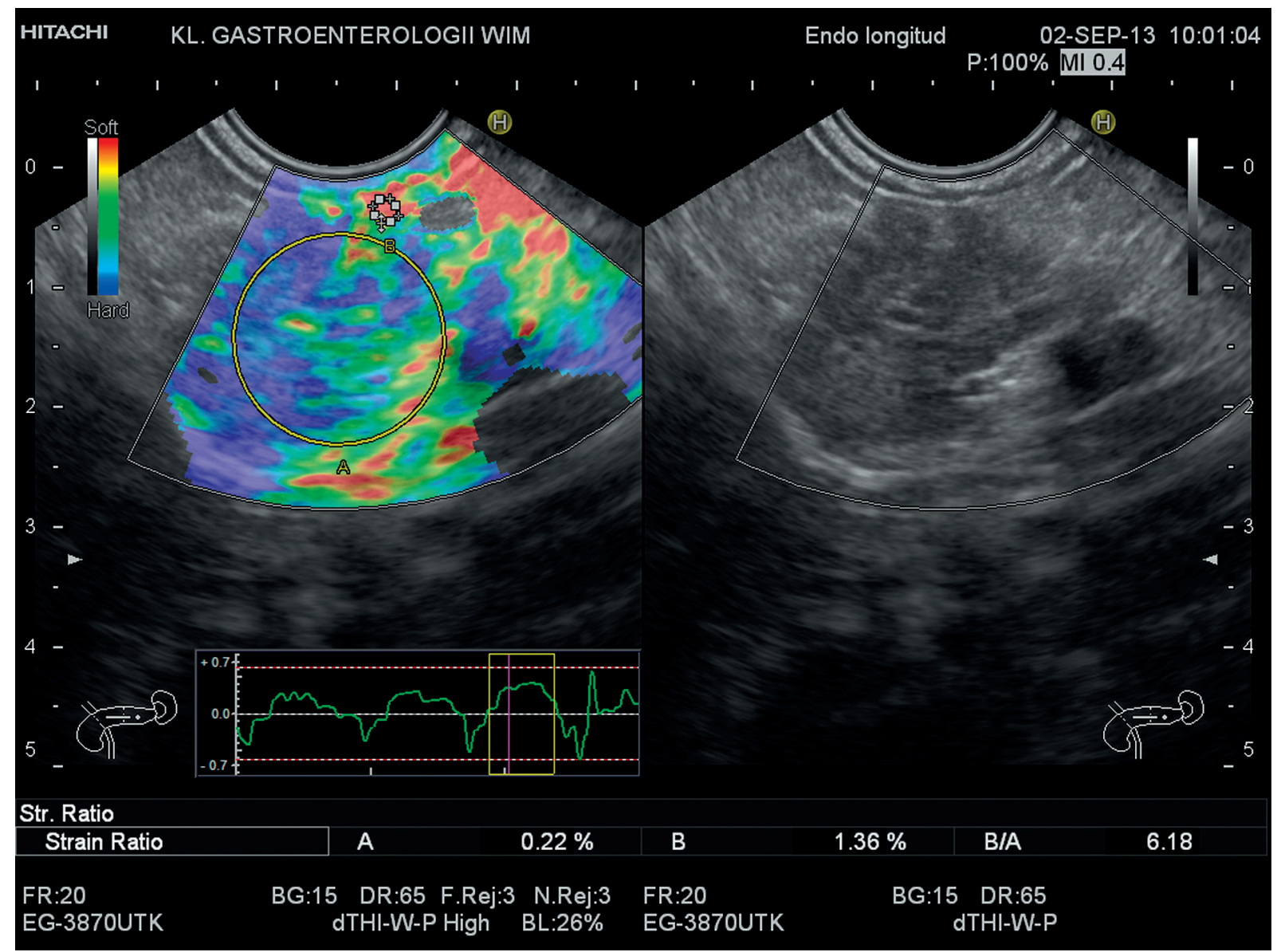

Figure 2. Chronic inflammatory process of the pancreas in elastography

is the one by Iglesias-Garcia et al. The study is based on quantitative analysis software examining the elasticity of the tissue. Iglesias-Garcia et al. examined the elasticity of pancreatic tumours and the strain ratio $(\mathrm{SR})$ in all cases. Strain ratio is defined as the relation between the elasticity of normal tissue (reference) and the elasticity of the interest area (ROI). The sensitivity of this study with quantitative analysis was $100 \%$, and the specificity was about 93\% [17].

In the current study we examined the elasticity of the pathological pancreas tissue and the strain ratio. In normal pancreatic tissue the average elasticity was $0.54 \%$ (Figure 1 ), and in inflammatory lesions it was $0.26 \%$ (Figure 2). The average elasticity for adenocarcinoma tumours, neuroendocrine tumours, and squamous cell carcinomas was 0.025 (Figure 3). The strain ratio factor was observed in inverse proportion and was the lowest in normal pancreatic tissue (average 1.79), while it was higher in inflammatory tumours (5.35). The highest value of the strain ratio factor was in adenocarcinoma tumours, neuroendocrine tumours, and squamous cell carcinomas (33.93). We observed that the strain ratio factor was much higher in malignant lesions than in inflammatory ones and in normal pancreatic tissue. The elasticity parameters were the highest in normal pancreatic tissue, lower in inflammatory tumours, and the lowest in malignant tumours. The elastography with the strain ratio and elasticity parameters valuation proved to be helpful in the differentiation of pancreatic solid tumours. However, more studies are needed to define the usefulness of this method in diagnosing solid pancreatic tumours.

\section{Conclusions}

Differential diagnosis of chronic pancreatitis and pancreatic cancer remains one of the most difficult diagnostic challenges. Endosonography is the best method in diagnosing pancreatic solid tumours due to its high sensitivity and specificity when combined with FNA biopsy and elastography. The strain ratio factor reaches much higher values for malignant tumours than in the case of inflammatory tumours and normal pancreatic tissue. The elasticity parameters are the highest in normal pancreatic tissue, lower in inflammatory tumours, and the lowest in malignant tumours. 


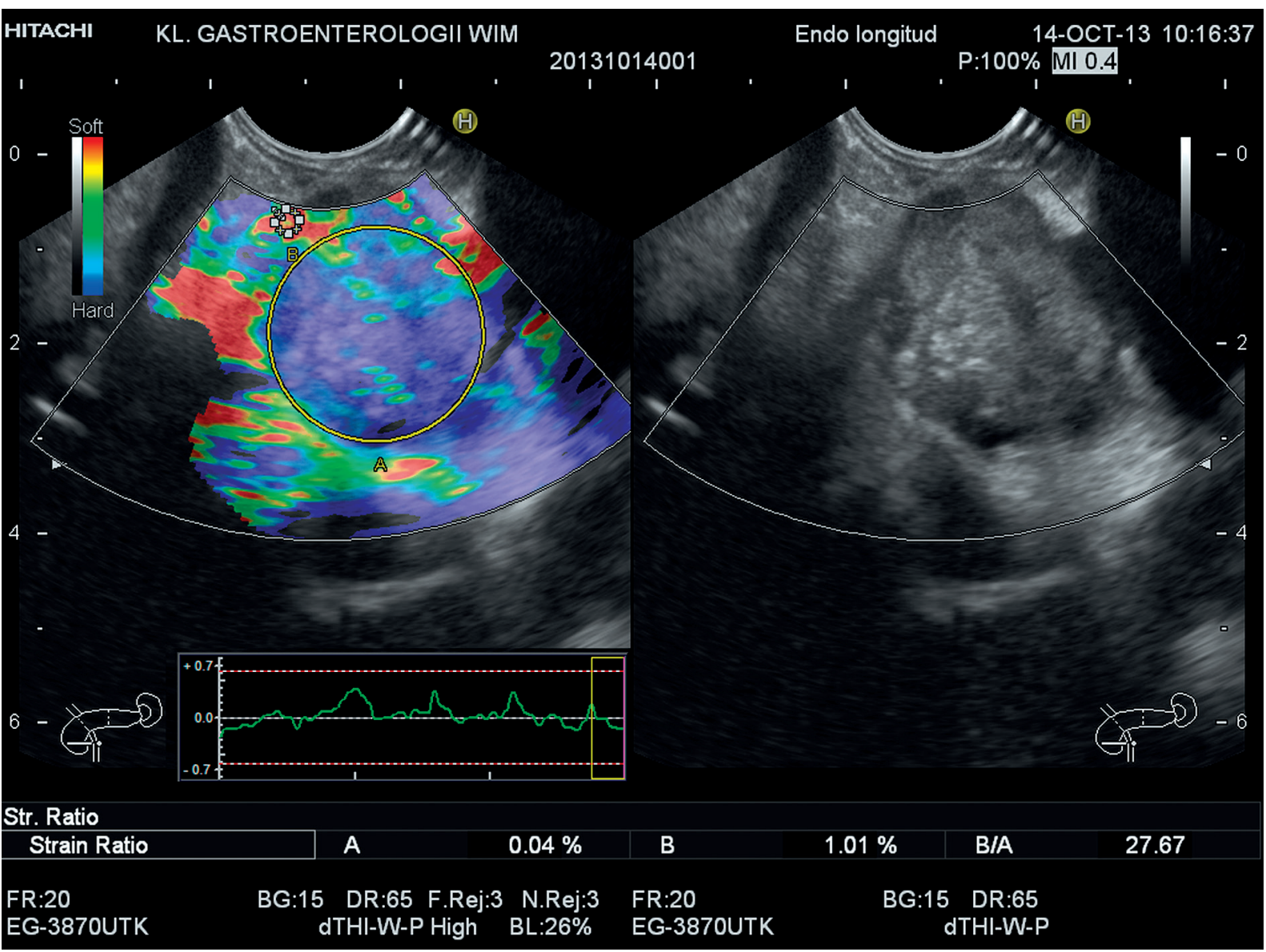

Figure 3. Pancreatic cancer in elastography

\section{Conflict of interest}

The authors declare no conflict of interest.

\section{References}

1. Cascinu S, Falconi M, Valentini V, et al. Pancreatic cancer: ESMO Clinical Practice Guidelines for diagnosis, treatment and follow-up. Ann Oncol 2010; 21: 55-8.

2. Seufferlein T, Bachet JB, Van Cutsem E, et al. Pancreatic adenocarcinoma: ESMO-ESDO Clinical Practice Guidelinesfor diagnosis, treatment and follow-up. Ann Oncol 2012; 23: 33-40.

3. Sharma C, Eltawil KM, Renfrew PD, et al. Advances in diagnosis, treatment and palliation of pancreatic carcinoma: 19902010. World I Gastroenterol 2011; 17: 867-97.

4. Giovannini M. Contrast-enhanced endoscopic ultrasound and elastosonoendoscopy. Best Pract Res Clin Gastroenterol 2009, 23: 767-79.

5. Giovannini M, Thomas B, Erwan B, et al. Endoscopic ultrasound elastography for evaluation of lymph nodes and pancreatic masses: a multicenter study. World J Gastroenterol 2009; 15: 1587-93.

6. Itoh A, Ueno E, Tohno E, et al. Breast disease: clinical application of US elastography for diagnosis. Radiology 2006; 239: 341-50.
7. Saftoiu A, Vilman P. Endoscopic ultrasound elastography a new imaging technique for the visualization of tissue elasticity distribution. J Gastrointest Liver Dis 2006; 15: 161-5.

8. Harewood GC, Wiersema MJ. Endosonography-guided fine needle aspiration biopsy in the evaluation of pancreatic masses. Am J Gastroenterol 2002; 97: 1386-91.

9. Varadarajulu S, Tamhane A, Eloubeidi MA. Yield of EUS-guided FNA of pancreatic masses in the presence or the absence of chronic pancreatitis. Gastrointest Endosc 2005; 62: 728-36.

10. Chang KJ, Nguyen P, Erickson RA, et al. The clinical utility of endoscopic ultrasound-guided fine-needle aspiration in the diagnosis and staging of pancreatic carcinoma. Gastrointest Endosc 1997; 45: 387-93.

11. Iglesias García J, Domínguez-Muñoz JE. Endoscopic ultrasound guided biopsy for the evaluation of pancreatic tumors. Gastroenterol Hepatol 2007; 30: 597-601.

12. Saftoiu A, Vilmann P, Gorunescu F, et al. Neural network analysis of dynamic sequences of EUS elastography used for the differential diagnosis of chronic pancreatitis and pancreatic cancer. Gastrointest Endosc 2008; 68: 1086-94.

13. Iglesias-Garcia J, Larino-Noia J, Abdulkader I, et al. EUS elastography for the characterization of solid pancreatic masses. Gastrointest Endosc 2009; 70: 1101-8. 
14. Hirche TO, Ignee A, Barreiros AP, et al. Indications and limitations of endoscopic ultrasound elastography for evaluation of focal pancreatic lesions. Endoscopy 2008; 40: 910-7.

15. Janssen J, Schlörer E, Greiner L. EUS elastography of the pancreas: feasibility and pattern description of the normal pancreas, chronic pancreatitis, and focal pancreatic lesions. Gastrointest Endosc 2007; 65: 971-8.

16. Fritscher-Ravens A. Blue clouds and green clouds: virtual biopsy via EUS elastography? Endoscopy 2006; 38: 416-7.

17. Iglesias-Garcia J, Larino-Noia J, Abdulkader I, et al. Quantitative endoscopic ultrasound elastography: an accurate method for the differentiation of solid pancreatic masses. Gastroenterology 2010; 139: 1172-80.

Received: 12.06 .2014

Accepted: 5.09.2014 